\title{
Aplicación de estrategias virtuales para mejorar el desarrollo del pensamiento lógico en matemáticas
}

\author{
Application of virtual strategies to improve the development of logical thinking in \\ mathematics
}

Estrategias virtuales en el desarrollo del pensamiento

\author{
María Vanessa Pibaque Cedeño Mg ${ }^{(1)}$ \\ Carlina Vélez Villavicencio $\mathbf{P h D}{ }^{(2)}$ \\ ${ }^{1}$ Universidad San Gregorio de Portoviejo. Ecuador. email: e.mvpibaque@ sangregorio.edu.ec \\ ${ }^{2}$ Universidad San Gregorio de Portoviejo. Ecuador. email: cevelez@ sangregorio.edu.ec
}

Contacto: e.mvpibaque@ sangregorio.edu.ec

\section{Resumen}

La presente investigación, tiene como objetivo determinar las estrategias virtuales en el área de matemática que aportan al desarrollo del pensamiento lógico en los estudiantes para lograr la mejora de las habilidades y destrezas cognitivas y fortaleciendo el proceso de enseñanza aprendizaje. Este trabajo investigativo, tuvo un enfoque de tipo cuali- cuantitativo, que se enmarcó en la investigación: exploratoria, descriptiva, explicativa, bibliográfica, para su análisis se utilizaron los métodos inductivo, deductivo, analítico y sintético, por otro lado, se utilizó la técnica de recolección de información primaria a través de encuestas aplicadas a los estudiantes y docentes, además se ejecutó la entrevista a un experto en el área de matemática. Con el desarrollo de este estudio, se fortalecen los conocimientos en la aplicación de las estrategias virtuales en el desarrollo del pensamiento lógico de las matemáticas. En base a los resultados se logró identificar que los docentes utilizan estrategias tradicionales en las clases, con poco uso de la tecnología, la deficiente aplicación de estrategias virtuales creativas afecta en el desarrollo del pensamiento lógico del grupo de estudiantes en estos tiempos de confinamiento. Por consiguiente, se concluye que es importante la implementación de 
aplicaciones virtuales, porque mejora positivamente el pensamiento lógico en el área de matemáticas con la aplicación correcta y eficiente de dichas estrategias virtuales, y así dinamizar el proceso educativo y aportar significativamente en el desarrollo de las habilidades y destrezas de los estudiantes.

Palabras clave: Creatividad: enseñanza aprendizaje: estrategias virtuales: herramientas virtuales: pensamiento lógico.

\begin{abstract}
The objective of this research is to determine the virtual strategies in the area of mathematics that contribute to the development of logical thinking in students to achieve the improvement of cognitive abilities and skills and strengthening the teaching-learning process. This research work had a qualitative-quantitative approach, which was framed in the research: explorative, descriptive, explanatory, bibliographic, for its analysis the inductive, deductive, analytical and synthetic methods were used, on the other hand, the primary information collection technique through surveys applied to students and teachers, in addition, an interview was carried out with an expert in the area of mathematics. With the development of this study, knowledge in the application of virtual strategies in the development of logical thinking in mathematics is strengthened. Based on the results, it was possible to identify that teachers use traditional strategies in classes, with little use of technology, the deficient application of creative virtual strategies directly affects the development of logical thinking of students in these times of confinement. Therefore, it is concluded that the implementation of virtual applications is important, because it positively improves logical thinking in the area of mathematics with the correct and efficient application of these virtual strategies, and thus dynamize the educational process and contribute significantly to the development of the abilities and skills of students.
\end{abstract}

Keywords: Creativity: teaching learning: virtual strategies: virtual tools: logical thinking.

\title{
Introducción
}

El rol de los docentes, juega un papel fundamental a la hora de generar estrategias virtuales creativas en el aprendizaje. Es así que estos, deben ser guías y facilitadores del proceso de enseñanza aprendizaje, de modo que deben cumplir con las necesidades y exigencias que se puedan presentar los estudiantes al desarrollar el pensamiento lógico matemático. 
En los actuales momentos, se genera un problema que con el tiempo sigue tomando fuerza y los estudiantes seguirán siendo los más perjudicados, ocasionando dificultades en sus estudios, debido al inadecuado aporte y uso de las estrategias virtuales creativas de parte de los docentes, lo cual se puede mejorar con la correcta utilización de las estrategias con el fin de no limitar el pensamiento lógico en la asignatura de matemáticas.

El proceso de los entornos virtuales que ayudan al aprendizaje, se lo puede visualizar con aquel espacio físico en donde no se cuenta con la infraestructura de paredes y por tanto, se lo conoce también como un espacio social, cuya herramienta primordial es el internet, este proceso no es presencial y es multicrónico, es decir; cuenta con redes electrónicas que son las llevarán al éxito a este tipo de espacios virtuales. (Bello, 2018, p. 22)

El problema, es notable y se ve reflejado en la institución, escenario de la investigación, donde los estudiantes no desarrollan el pensamiento lógico en la asignatura de matemáticas, por lo que es necesario la adecuada aplicación de estrategias didácticas creativas en el área de matemáticas de parte de los docentes y lograr aprendizajes significativos en los estudiantes, a partir de una participación activa, responsable e innovadora, utilizando estrategias didácticas creativas en la asignatura de matemáticas con el fin de lograr metas propuestas puedan desarrollar su pensamiento lógico en la resolución de problemas matemáticos.

Guzmán (2020) afirma que "el uso de las nuevas tecnologías en el proceso educativo es de gran utilidad, al ofrecer herramientas más acordes a las expectativas e intereses de cada uno de los alumnos, y así poder aportar en el desarrollo de los diversos contenidos que favorezcan el proceso de enseñanza aprendizaje" (p. 34). Por ende, en esta investigación, se pretende analizar y establecer estrategias virtuales creativas que contribuyan a una vinculación más activa de los recursos tecnológicos al desarrollo de las clases en matemáticas, esta área aporta al desarrollo cognitivo de los educandos, sea presencial o de manera virtual el docente debe aplicar estrategias metodológicas para el mejor desempeño de los educandos.

En base a los procesos teóricos, se formula el problema: limitada aplicación de las estrategias virtuales creativas en el área de matemáticas para el aporte del desarrollo del pensamiento lógico en los estudiantes, se ha observado que los docentes no aplican este tipo de estrategias para mejorar el aprendizaje creativo en sus estudiantes, lo que perjudica su aprendizaje de manera directa, es decir, 
ellos aún siguen siendo partícipes de una educación totalmente tradicional y por ello, no se logran alcanzar los aprendizajes requeridos en los estudiantes.

Ante esto, se expone también los resultados que emite el Instituto Nacional de Evaluación Educativa del Ecuador, en su informe de resultados Ser Bachiller del año 2016 - 2017, en donde se evaluaron a 265083 estudiantes, de los cuales en el dominio matemático el 32,4\% reflejó una calificación deficiente, el 43,4\% elemental, el 23,0\% satisfactorio y el 1,2\% excelente. (INEVAL, 2017)

Desde aquí surge una interrogante que es fundamental en el desarrollo de este proceso investigativo: ¿Cómo la adecuada aplicación de las estrategias virtuales creativas en el área de matemática, aporta significativamente en el desarrollo del pensamiento lógico de los estudiantes?, y dada la importancia y relevancia del tema, se da paso al análisis de las experiencias de los docentes que proporcionan resultados verídicos en este proceso investigativo.

Es fundamental, que se desarrollen entornos virtuales aptos para favorecer el aprendizaje de los estudiantes en el área de matemáticas, y que sea el docente quien aplique una diversa gama de actividades que ayuden a mejorarla desde la perspectiva del desarrollo de aprendizaje. Pérez (2017) menciona que "se tienen que desarrollar ambientes virtuales, dado que es aquí donde por medio del uso de la tecnología se logrará facilitar la comunicación y procesamiento de la información; logrando que el estudiante se relacione con el conocimiento y consigo mismo" (p. 12). Se puede señalar que las herramientas para mejorar las clases de matemáticas se encuentran divididas en algunos grupos, dadas por funciones y gráficas, videos, juegos y actividades interactivas, matemática práctica. En este trabajo, se detalla como objetivo principal determinar las estrategias virtuales en el área matemática que aportan al desarrollo del pensamiento lógico en los estudiantes.

El proceso educativo, debe estar orientado hacia el propósito de los cambios que se proyecten en este medio, por ello; se debes propiciar espacios aptos que ayuden en la formación de los estudiantes, con el que se logren incorporar innovadoras tecnologías, las cuales conducen a propiciar un mejor aprendizaje en las aulas y con una perspectiva de poder favorecer los aprendizajes y así facilitar los medios que sustenten el desenvolvimiento de desarrollo de conocimientos. (Linares, 2019, p. 22). 
La implementación tecnológica virtual en la educación, ha permitido optimizar el proceso de enseñanza- aprendizaje situación que es evidenciada todos los días en la práctica del docente, sin embargo, conduce a nuevos planteamientos al momento de enseñar. Romero (2020) afirma que:

En el aprendizaje de las matemáticas se pueden utilizar actividades lúdicas y dinámicas para satisfacer las necesidades del estudiante actual, las estrategias virtuales creativas se convirtieron en pilar fundamental en el ámbito educativo, donde existe variedades de actividades que se pueden aplicar de manera virtual para desarrollar el pensamiento lógico matemático, siendo además de gran utilidad para potenciar los conocimientos en la enseñanza y aprendizaje. (p. 23)

Por lo tanto, se puede decir que, un entorno virtual de aprendizaje aplicando estrategias didácticas, lúdicas e interactivas se les permiten a los participantes la construcción del conocimiento, la cooperación y un buen nivel de aprendizaje en los estudiantes.

Las TIC, son una herramienta que facilitan el proceso de enseñanza y aprendizaje de las matemáticas, pero su uso en el aula requiere una metodología adecuada, un cambio metodológico notable. Los docentes, deben cumplir con su rol de una manera fundamental en los diversos episodios que se les presenten en el espacio educativo, es por ello necesario que se aprovechen cada una de las herramientas con las que se cuenta para fortalecer el proceso de enseñanza aprendizaje, es necesario también que se incorpore a las tic en la enseñanza y que este sea un espacio eficiente para el aporte en la adquisición de las habilidades y destrezas. (Molina, 2019, p. 22)

En el Ecuador, con los procesos actuales vividos, poco a poco se está implementando estrategias virtuales que aportan como recursos en el ámbito de aprendizaje y por ende desarrolla el pensamiento lógico matemático, cabe señalar que las tics han aportado significativamente en el proceso de enseñanza aprendizaje, y esto se ha logrado evidenciar en todos los tiempos desde que la tecnología ha comenzado a ser parte del entorno social educativo de los estudiantes.

Es necesario conocer aspectos importantes que hacen relevancia en este trabajo investigativo, entre ellos está el tema del pensamiento lógico, que se concreta siendo toda aquella acción, que sirve para el razonamiento y que en realidad la realiza el ser humano para concretar actividades diarias de su accionar, de esta manera se concreta que dichas relaciones logran el involucramiento de objetos que son reales y abstractos, y, por ende, existe una relación directa entre cada uno de ellos. 
Según Travieso (2018) se debe realizar un enfoque entre el vínculo del pensamiento lógico y el proceso de enseñanza aprendizaje, ya que los estudiantes deben apropiarse de los niveles de desarrollo en base a sus pensamiento, de tal manera que se logre articular las ideas y se concreten en aspectos que propician un desarrollo adecuada de las habilidades y destrezas de matemáticas.

Las estrategias virtuales se consideran como un conjunto de elementos que están elaborados con la finalidad de facilitar el proceso educativo en entornos virutales, según Camacho (2018) afirma que las estrategias, son un recurso que permiten a los estudiantes conocer sobre los temas y comprender lo que se estudia, por ende también, mejoran la motivación del aprendizaje de los estudiantes (p. 4). Entonces, cabe señalar que en los actuales momentos, es necesario que el docente busque e indague en su propio rol, que participe con estrategias innovadoras que aporten a la enseñanza aprendizaje, para llegar a aplicar las mejores estrategias en el espacio virtual en el que actualmente se desenvuelve, de tal manera que se logren alcanzar los objetivos propuestos en su entorno de aprendizaje actual.

\section{Materiales y métodos}

Esta investigación, consideró un enfoque cualitativo cuantitativo, donde indagó conceptualizaciones de las estrategias virtuales creativas y su aporte al desarrollo del pensamiento lógico de los estudiantes en la asignatura de matemáticas. El proyecto de investigación, se enmarcó en los tipos de investigación: exploratoria, descriptiva, explicativa, bibliográfica, y prospectiva, los cuales se utilizaron a lo largo de la investigación, donde se exploró e indagó información del tema de estudio, logrando interpretar los resultados obtenidos e indicar puntos de vistas favorables en el proceso de enseñanza aprendizaje.

Los métodos Inductivo - Deductivo y Analítico - Sintético se utilizaron en la presente investigación. Las técnicas de recolección de información primaria utilizada, fueron las encuestas a los estudiantes y docentes de la Unidad Educativa Fiscal "El Anegado" en el cantón Jipijapa, Ecuador. La entrevista a un experto del área de matemática contribuyó a reafirmar la problemática y recomendaciones de mejora.

Este trabajo, se realizó con una población total de 104 estudiantes, 25 docentes y 1 experto, el instrumento para la recolección de información, fue el cuestionario de base estructurada, para la obtener la información de parte de los estudiantes y docentes, con el fin de plantear los resultados para analizarlos mediante el método estadístico.

\section{Resultados}


Los resultados son emitidos, en base a la entrevista y encuesta aplicadas en este proceso investigativo: Los docentes ocasionalmente organizan estrategias virtuales creativas que permitan el desarrollo del pensamiento lógico matemático en los estudiantes de manera ocasional el 44\%, interpretando que los docentes realizan una organización de estrategias virtuales para el desarrollo del pensamiento lógico en sus estudiantes.

Para Villamil (2018) hace conocer que "para que se logre alcanzar el desarrollo del pensamiento lógico de matemática, se deben proyectar estrategias adecuadas que propicien un buen aprendizaje en los estudiantes, motivándoles a aprender y que muestren interés por esta área” (p. 22).

Los docentes utilizan estrategias virtuales para el proceso de enseñanza aprendizaje en el área de matemáticas algunas veces 41,35\%. En referencia a estos aspectos Huertas (2018) menciona que los docentes, han tenido que buscar medidas diversas, son alternativas que ayuden a favorecer el proceso de enseñanza aprendizaje, incluyendo estrategias que brinden soporte en línea, que ayuden al proceso multidisciplinar en el que se integre el software matemático y estadístico. De esta manera los docentes, deben ser innovadores y creativos ya que, en la educación presencial, el desarrollo de habilidades matemáticas es una tarea compleja tanto para los profesores como para los alumnos.

El entrevistado, indica que los docentes viven siempre avanzando y mejorando varias estrategias, muchos conocimientos, por lo que considera que es importante el uso y manejo de estrategias virtuales para desarrollar el pensamiento lógico en la asignatura de matemáticas, para la adquisición de conocimientos y dominio moderno del aprendizaje. En tal virtud, se puede indicar que las estrategias virtuales toman importancia en su uso y aplican en la actualidad en la asignatura de matemáticas, logrando utilizar herramientas virtuales que favorezcan el conocimiento del estudiante.

Para Jiménez (2019) menciona que el buen uso de la tecnología aporta de manera significativa al proceso de desarrollo del pensamiento lógico matemático de los estudiantes, es así que favorece y estimula la búsqueda de soluciones de los problemas que se suelen presentar y permiten la participación activa de los alumnos.

Los resultados, evidencian que existe un $84 \%$ en nivel medio el desarrollo del pensamiento lógico de los estudiantes (Tabla 1). De esta manera, se establece que los procesos de enseñanza aprendizaje se llevan de manera incorrecta, donde la utilización de estrategias virtuales creativas de parte de los docentes es necesaria para mejorar el pensamiento lógico, puesto que los docentes no utilizan las herramientas indispensables en su tiempo de trabajo. 
Está demostrado que poseer un pensamiento lógico desarrollado altamente facilita la resolución de los problemas matemáticos, siempre y cuando se utilicen correctamente, puesto que existen docentes que no saben aplicarlo para la resolución de problemas matemáticos, por tanto, se hace indispensable en ocasiones adquirir el conocimiento específico para llevar a cabo una correcta resolución. (Véliz, 2018, p. 23)

\section{Tabla 1}

El nivel de desarrollo del pensamiento lógico de los estudiantes

\begin{tabular}{lc}
\hline \multicolumn{1}{c}{ ALTERNATIVAS } & Porcentajes \\
\hline Alto & 4,00 \\
Medio & 84,00 \\
Bajo & 12,00 \\
\hline Total & $\mathbf{1 0 0 , 0 0}$ \\
\hline
\end{tabular}

El entrevistado, indica que las instituciones deben fortalecer los conocimientos de los docentes mediante vínculos externos o internos para ser partícipes de capacitaciones constantes más aún para dominar todas las herramientas o plataformas virtuales, las cuales ayudan en el proceso de aprendizaje de los estudiantes. Por tanto, se puede indicar que las plataformas educativas virtuales ayudan significativamente a aprender y comprender los diferentes temarios en el área de matemática, de una manera creativa, innovadora y utilizando recursos tecnológicos que motivan e integran al estudiante. Según Jiménez (2019) afirma en la enseñanza de las matemáticas, el docente debe promover experiencias que permitan articular los contenidos, al que se hace necesario que el docente ofrezca nuevas orientaciones en su quehacer pedagógico, incorporando nuevas herramientas de trabajo, por ejemplo, las llamadas herramientas de la informática y la comunicación - TICS.

Se considera que la implementación de este tipo de estrategias ha sido utilizada muy poco, pero, sin embargo, ha ayudado en la actualidad ante la situación que se está viviendo debido la pandemia a nivel mundial, donde las estrategias virtuales han aportado significativamente en el proceso de enseñanza aprendizaje, logrando motivar e integrar a todos los estudiantes. Al respecto, las herramientas digitales, en el proceso de enseñanza aprendizaje ayudan a proveer, fortalecer el proceso de enseñanza aprendizaje de las matemáticas. Rodríguez (2013) hace referencia a que los maestros, deberían tener en cuenta las mejores prácticas para enseñar matemática y se recomienda la integración 
de las tecnologías de la información y la comunicación (TICs) en los programas escolares de Matemática.

El entrevistado, indica que existen varias estrategias virtuales tales como Microsoft teams, zoom, Educaplay, Kahoot, las cuales son actualizadas, gratis según los accesos, de fácil manejo e interesantes donde los estudiantes participan de manera activa, asíncrona, síncrona, operacional y de manera interactiva específicamente en el área de matemáticas desarrollando destrezas y habilidades en los estudiantes. De lo que se puede indicar, que existen estrategias virtuales que se pueden utilizar de manera creativa, integradora y didáctica con el fin de desarrollar el pensamiento lógico de los estudiantes.

Al respeto, Díaz (2018) indica "que una de las alternativas que existen en la actualidad es la utilización de la tecnología que ofrece una gama de herramientas para disímiles actividades de la vida diaria" (p. 28). Las cuales facilitan una serie de medios que se pueden valer para el trabajo docente y como estrategia de enseñanza-aprendizaje para desarrollo del pensamiento lógico matemático del estudiante.

\section{Discusión}

En relación a los datos obtenidos en los instrumentos aplicados, se va a iniciar con la encuesta realizada a los docentes donde se logró determinar que el $44 \%$ de los docentes organizan estrategias virtuales de manera ocasional, por lo que es necesario que los docentes apliquen estrategias virtuales todos los días para mejorar continuamente el desempeño y el rendimiento educativo del alumno, conllevando a desarrollar el pensamiento lógico matemático.

Según lo señalado por Coll (2019) las estrategias virtuales influyen en el aprendizaje o el rendimiento de los alumnos hacia el interés por estudiar matemáticas, así como se insertan en las prácticas educativas y cómo, eventualmente, pueden transformarlas y mejorarlas, asumiendo que el aprendizaje de los alumnos se relaciona, y depende de la calidad y aplicación frecuente dentro del aula. De esta manera los docentes deben innovar y aplicar nuevas estrategias virtuales creativas en todas las clases con el fin de fortalecer los conocimientos de los educandos.

También, se evidencia en los resultados que el $48 \%$ de docentes nunca emplea aplicaciones web como estrategias virtuales en el área de matemáticas, afirmando el 56\% de los docentes que se les resulta difícil su implementación, de allí que se considera que existe poca utilización de las estrategias virtuales de parte de los docentes a los estudiantes, pero cuando utilizan las estrategias virtuales el 
$68 \%$ de los estudiantes demuestra interés en el proceso de enseñanza aprendizaje de manera permanente.

De esta manera, se puede evidenciar que las estrategias virtuales ayudan a los estudiantes a mostrar interés y dedicación en los procesos de aprendizaje en el área de matemáticas. Al respecto, coincide con lo señalado por donde indica que las estrategias virtuales son utilizadas para mejorar el proceso de enseñanza-aprendizaje, promover el desarrollo de habilidades interpersonales, complementar la educación presencial y facilitar el seguimiento del aprendizaje logrando calidad educativa del aprendizaje y la motivación.

Además, los docentes afirman que el $84 \%$ de los educandos tiene el desarrollo del pensamiento lógico en nivel medio y los estudiantes evidencian que el $41.35 \%$ de los docentes utiliza la aplicación Excel, pero las demás herramientas virtuales no son aplicadas por los docentes de forma frecuente, tal es el caso de Quiziz, Puzzles, Kahoot, geogebra y graspable math que tienen un porcentaje entre 44,23\% y $46,15 \%$, conlleva de esta manera a limitar el pensamiento lógico en los estudiantes.

Al respecto, Huamaní, (2017) indica que las herramientas y materiales tecnológicas del área de matemáticas facilitan el desarrollo de distintas habilidades, estilos y ritmos de aprendizaje por parte de los estudiantes y a los docentes le permiten generar propuestas metodológicas innovadoras y creativas para mejorar la cognición y el proceso de aprendizaje.

Las autoras sistematizan los aportes de esta investigación, al estudiar las estrategias virtuales identificadas tanto por los autores consultados como los actores objeto del estudio; se presentan con su respectivo aporte al desarrollo del pensamiento lógico matemático, según la tabla 2.

Tabla 2.

Aportes de las estrategias virtuales en el desarrollo el pensamiento lógico matemático:

\begin{tabular}{|l|l|}
\hline \multicolumn{1}{|c|}{ Estrategias virtuales } & \multicolumn{1}{|c|}{ Aportes al pensamiento lógico matemático } \\
\hline Quiziz & Aporta al conocimiento y al desarrollo intelectual en los niños \\
\hline Puzzles & $\begin{array}{l}\text { Contribuye a la motricidad fina y mejora las habilidades de } \\
\text { ordenamiento lógico. }\end{array}$ \\
\hline Kahoot & Genera procesos de concentración y de interacción \\
\hline Geogebra & Atiende al desarrollo del pensamiento racional \\
\hline Graspable math & $\begin{array}{l}\text { Aporta al desarrollo del pensamiento relacional y concreto porque se } \\
\text { aprende haciendo. }\end{array}$ \\
\hline
\end{tabular}


Estas explicaciones también dan atención a la entrevista realizada al experto en la que se logra determinar que la utilización e implementación de estrategias virtuales aportan al desarrollo del pensamiento lógico matemático, puesto que fomenta el interés, la creatividad y motivación en el área de matemáticas. Coincidiendo con Vaca (2020) se debe implementar situaciones didácticas diferentes para que exista una interacción que permitan el avance del pensamiento lógico matemático por medio de la complejidad de diferentes ejercicios propuestos tomando como apoyo directo las estrategias virtuales creativas.

\section{Conclusiones}

El estudio teórico de campo, permite identificar que los docentes utilizan estrategias tradicionales en las clases tales como la exposición del docente con poco uso de la tecnología y las estrategias virtuales son aplicadas ocasionalmente en la asignatura de matemáticas, por lo que es necesario su uso y desarrollo como mecanismo de comunicación, seguimiento, integración, motivación y evaluación, dentro del espacio en el cual donde los protagonistas principales son los docentes y estudiantes con el fin de desarrollar el empeño y dedicación en el aprendizaje.

El desarrollo del pensamiento lógico de los estudiantes, se encuentra en un nivel medio según los docentes y estudiantes debido a la utilización de procesos repetitivos y a la falta de aplicación de estrategias virtuales, aunque existen de forma gratuita varias aplicaciones virtuales, la dificultad al utilizarlo, hace que los docentes no lo apliquen correctamente dentro del proceso de enseñanza aprendizaje en el área de matemáticas para el desarrollo del pensamiento lógico, por tanto, es preciso desarrollar de manera secuencial y diariamente este pensamiento, donde los estudiantes serán capaces de converger la hipótesis, la síntesis, la interpretación y el análisis.

Las aplicaciones virtuales Quizizz, Puzzles, Kahoot, Geogebra, Graspable Math, y Excel se perciben como idóneas para el desarrollo del pensamiento lógico en el área de matemáticas, con su aplicación correcta y de manera frecuente dinamizando el proceso educativo e incluyendo además juegos virtuales interactivos, video de la explicación de clases y ejercicios resueltos potenciando la aplicación de mensajería instantánea - WhatsApp, lo cual favorece el rendimiento académico e interés por el aprendizaje.

\section{Referencias bibliográficas}

Bello E. (2018). Educación virtual: Aulas sin paredes. Revista Iberoamericana, 22. Obtenido de https://educrea.cl/educacion-virtual-aulas-sin-paredes/ 
Camacho G. (2018). Estrategias de aprendizajes para entornos virtuales. Redalyc, 4.

Coll S. (2019). Los entornos virtuales como espacios de enseñanza y aprendizaje. Revista Iberoamericana, $56 . \quad$ Obtenido de http://www.scielo.org.mx/scielo.php?script=sci_arttext\&pid=S1405-66662010000100009

Díaz J. (2018). Aprendizaje de las matemáticas con el uso de simulación. Scielo , 28. Obtenido de http://www.scielo.org.co/scielo.php?script=sci_arttext\&pid=S1794-89322018000100022

Guzmán C. (2020). La educación virtual: logros alcanzados y nuevos desafíos. España: Paidos. Obtenido de https://www.evaluacion.gob.ec/wpcontent/uploads/downloads/2019/02/CIE_ResultadosEducativos18_20190109.pdf

Huertas M. (2018). Aprendizaje virtual de las matemáticas. España: Paidos. Obtenido de https://rusc.uoc.edu/rusc/ca/index.php/rusc/article/download/v9n1-aprendizaje-virtualmatematicas/1431-3069-1-PB.pdf

INEVAL. (2017). Informe de resultados Ser Bachiller 2016 - 2017. Quito: Ineval. Obtenido de https://cloud.evaluacion.gob.ec/dagireportes/nacional/2016-2017.pdf

Jiménez C. (2019). Enseñanza de las matemáticas básicas en un entorno e-Learning: un estudio de caso de la Universidad Manuela Beltrán Virtual. Redalyc, 78. Obtenido de http://www.scielo.org.co/scielo.php?script=sci_arttext\&pid=S0120-81602015000200011

Jiménez D. (2019). Herramientas digitales para la enseñanza de las matemáticas en la educación. Scielo 24.

Obtenido

de https://repository.ucc.edu.co/bitstream/20.500.12494/11110/1/2019_herramientas_digitales_ matematicas.pdf

Linares C. (2019). Los retos del sistema educativo. Dialnet, 22.

Molina P. (2019). Las tic en la enseñanza de las matematicas. Scielo, 22. Obtenido de https://es.slideshare.net/PaoMol/las-tic-en-la-enseanza-de-las-matematicas 
Pérez H. (2017). Los entornos virtuales educativos. México: Trillas . Obtenido de https://www.eumed.net/rev/atlante/2017/01/matematica.html

Rodríguez E. (2013). Efectos del EduBlog. España: Punto Rojo . Obtenido de http://espacio.uned.es/fez/eserv/bibliuned:masterComEdred-Erodriguez/Documento.pdf

Romero C. (2020). El Razonamiento lógico. Ciencia y Educación , 23. Obtenido de http://repositorio.ug.edu.ec/bitstream/redug/50519/1/ROMERO\%20CASTRO\%20SARASU\%c3\%81REZ\%20MEN\%c3\%89NDEZ\%20ALEXANDRA.pdf

Travieso D. (2018). El desarrollo del pensamiento lgico en los estudiantes. Scielo, 33.

Vaca A. (2020). Entorno Virtual de Aprendizaje para fortalecer el razonamiento físico matemático mediante herramientasWeb 2.0en 3BGU. México: Trillas. Obtenido de http://repositorio.uisrael.edu.ec/bitstream/47000/2505/1/UISRAEL-EC-MASTER-EDU378.242-2020-050.pdf

Véliz G. (2018). Niveles de pensamiento y resolución de problemas matemáticos. Dialnet, 23. Obtenido de https://www.google.com/url?sa=t\&rct=j\&q=\&esrc=s\&source=web\&cd=\&cad=rja \&uact=8\& ved=2ahUKEwi5us3h_ePwAhUMZzABHW2ND4QFjAAegQIAhAD\&url=https\%3A\%2F\%2Fdialnet.unirioja.es\%2Fdescarga $\% 2$ Farticulo\%2 F4729449.pdf\&usg=AOvVaw0Bwtx8m0Wr0hdC-cT9Yp_v

Villamil C. (2018). Estrategias metodológicas para el desarrollo de habiidades del pensamiento lógico matemático. Ciencias y educación, 22. Obtenido de https://dspace.ups.edu.ec/bitstream/123456789/16199/4/UPS-CT007852.pdf 